\title{
Störbeeinflussung von Neurostimulatoren durch Koagulationsgeräte basierend auf der Verkopplung über den menschlichen Körper
}

\author{
M. Schick and F. M. Landstorfer \\ Universität Stuttgart, Institut für Hochfrequenztechnik, Pfaffenwaldring 47, 70569 Stuttgart, Germany
}

\begin{abstract}
Zusammenfassung. Am Beispiel der Verkopplung von Koagulationsgeräten und implantierten Neurostimulatoren wird die gegenseitige Störbeeinflussung von medizinischen Geräten über den menschlichen Körper dargestellt. Für die Berechnungen dieses Szenarios werden die verwendeten Geräte charakterisiert und modelliert. Ebenso wird ein nach DIN 33402 Teil 2 entwickeltes Modell des menschlichen Körpers verwendet. Für dessen elektrische Eigenschaften werden in Abhängigkeit von der Frequenz gewichtete Mittelwerte aus mehreren relevanten Organen berücksichtigt. Bei den durchgeführten Untersuchungen werden verschiedene Parameter, wie die Implantationsart des Neurostimulators, der Verlauf und die Länge dessen Elektrode, der Koagulationspunkt am menschlichen Körper, die Frequenz, mit der koaguliert wird, und die Länge des Koagulatorkabels variiert, um so zu ,worst-case“-Aussagen zu gelangen. In den Ergebniskurven lassen sich Überhöhungen beobachten, die sich auf Resonanzeffekte z.B. auf dem Koagulatorkabel zurückführen lassen.
\end{abstract}

\section{Einleitung}

Der ständig steigende Einsatz elektronischer Apparate im medizinischen Bereich führt dazu, dass eventuelle Wechselwirkungen solcher Geräte zu einem ernsthaften Sicherheitsproblem werden. In besonderem Maße kritisch sind Geräte, die mit hochfrequenten Strömen arbeiten. Im Rahmen eines von der Deutschen Forschungsgemeinschaft geförderten Projekts werden eben diese Auswirkungen von verschiedenen Störquellen auf den betrieblichen Ablauf in Kliniken untersucht. Ein mögliches Störszenario im Operationssaal in der Klinik ist die Einkopplung eines HF-Chirurgiegeräts in einen Neurostimulator. Neurostimulatoren sind implantierte medizinische Geräte, die mittels elektrischer Impulse Regionen im menschlichen Gehirn anregen. Durch den

Correspondence to: M. Schick

(schick@ihf.uni-stuttgart.de)
Einfluss elektromagnetischer Felder kann es zu einer Fehlfunktion des Neurostimulators kommen. Geräte, die eine solche Beeinflussung verursachen können, sind zum Beispiel HF-Chirurgiegeräte, zu denen auch Koagulatoren zählen. Im Folgenden ist eine Untersuchung dieser geschilderten EMVProblematik beschrieben.

\section{Verwendetes Körpermodell}

Dem Einfluss des menschlichen Körpers kommt bei dieser Untersuchung eine wichtige Bedeutung $\mathrm{zu}$. Um diesen bei den Berechnungen berücksichtigen zu können, wurde ein vereinfachtes Körpermodell entwickelt, das den Rechenaufwand für diese komplexen Problemstellungen auf ein verträgliches Maß begrenzt (siehe Abb. 1). Das Modell ist nach den Abmessungen aus DIN 33402 Teil 2 (vgl. Deutsch Norm, 1986) entworfen und repräsentiert eine männliche Person zwischen 41 und 60 Jahren mit einem Perzentil von 50, d.h. dass $50 \%$ der für die Norm vermessenen Personen diese bzw. kleinere Maße besitzen. Nicht in der Norm enthaltene Größen wurden an Versuchspersonen bestimmt.

Das Modell HMLF wurde speziell für das verwendete Feldberechnungsprogramm FEKO (EM Software \& Systems, 2003) aus kanonischen Strukturen zusammengesetzt. Zur Beschreibung der elektrischen Eigenschaften des menschlichen Körpers wird dabei ein homogenes Dielektrikum verwendet. Für dessen Permittivität und Leitfähigkeit werden frequenzabhängige gewichtete Mittelwerte verwendet. Die hierbei berücksichtigten Gewebe mit dem verwendeten Prozentsatz nach Koritke und Sick (1988) sind in Tabelle 1 aufgeführt.

Weitere Gewebearten mit ähnlichen elektrischen Eigenschaften sind dabei mit in den oben aufgeführten Geweben zusammengefasst. Für die einzelnen Gewebearten lassen sich in Abhängigkeit von der Frequenz die gewünschten elektrischen Größen bestimmen (Gabriel (1996) und Italian National Research Council (1997)). Der bei den Berechnungen verwendete Gesamtwert ergibt sich dann durch 
Tabelle 1. Berücksichtigte Gewebearten mit Gewichtungsprozentsatz.

\begin{tabular}{cc}
\hline Gewebe & Prozentsatz (in \%) \\
\hline Muskeln & 45 \\
Knochenmark & 24 \\
Lunge (eingeatmet) & 15 \\
Herz & 9 \\
Magen & 7 \\
\hline
\end{tabular}

Aufsummierung der einzelnen, mit ihrem Prozentsatz gewichteten Größen. Dieses Modell bietet nun die Möglichkeit, die angestrebten Untersuchungen durchzuführen.

\section{Charakterisierung der Störquelle: der Koagulator}

Ein Koagulator ist ein Hochfrequenzchirurgiegerät, das mit einem hochfrequenten Wechselstrom Gewebezellen zerteilt und gleichzeitig das Eiweiß in der Zelle verhärten lässt, also koaguliert. Durch diesen Effekt tritt keine Blutung auf, weswegen Koagulatoren heutzutage häufig eingesetzt werden. Durch den Stromfluss kann der Koagulator andere, den Patienten ebenfalls kontaktierende Geräte in ihrer Funktion beeinträchtigen. Von den beiden möglichen Betriebsarten wird hier der monopolare Fall betrachtet. Hierbei wird mit einer Elektrode zum Beispiel in Form eines Skalpells gearbeitet und eine großflächige Gegenelektrode auf dem Oberschenkel des Patienten angebracht. Durch den Stromfluss zwischen den beiden Elektroden im Gewebe des Patienten bzw. auf dessen Oberfläche kann es zu Beeinflussungen anderer Medizingeräte kommen.

Für die nachfolgenden Berechnungen ist vor allem die Kenntnis der Signalverläufe am Ausgang des Koagulators von Bedeutung. Bei den meisten Anwendungsfällen sind die Signalverläufe sinusförmig. Es gibt jedoch auch einige Fälle beim Koagulieren, bei denen eine in einem bestimmten zeitlichen Abstand neu angeregte, stark gedämpfte Sinusschwingung verwendet wird. Das zu diesem Signal gehörende Frequenzspektrum enthält relevante Anteile bis weit in den Megahertzbereich hinein, so dass in den durchgeführten Untersuchungen der betrachtete Frequenzbereich auf $100 \mathrm{kHz}$ bis $100 \mathrm{MHz}$ festgelegt ist.

In einer Voruntersuchung wurde der Einfluss der Isolierung der Schneideelektrode gesondert betrachtet. Vergleiche der elektromagnetischen Felder von Schneideelektroden mit und ohne Isolierung zeigen eine sehr gute Übereinstimmung, so dass bei den anschließenden Betrachtungen die Isolierung vernachlässigt wird.

\section{Charakterisierung der Störsenke: der Neurostimula- tor}

Als Störsenke wird ein Neurostimulator betrachtet, wie er zum Beispiel bei Parkinson- oder Epilepsie-Patienten zum

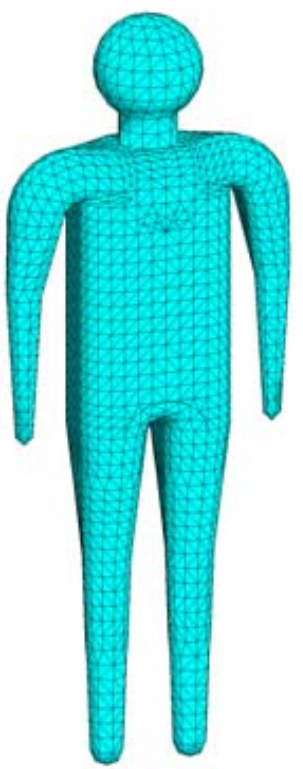

Abbildung 1. Entwickeltes Körpermodell HMLF.

Einsatz kommt. In einem Titangehäuse befindet sich eine Generatorschaltung zur Erzeugung eines hochfrequenten elektrischen Impulses, welcher die Nervenzellen im Gehirn stimuliert und so hirneigene, auf Grund von Krankheit fehlerhafte Impulse blockiert. In einer Voruntersuchung (Landstorfer et al., 1999) wurde der Einfluss verschiedener Formen des Gehäuses in den Berechnungen verglichen. Es wurden sowohl zwei- als auch dreidimensionale Gehäuse, sowie unterschiedliche Formen berücksichtigt. Da alle Gehäusemodelle nahezu dieselben Ergebnisse lieferten, wird für die hier dargestellte Untersuchung ein zweidimensionales Modell mit einer an den realen Stimulator angelehnte Form verwendet (siehe Abb. 2).

Das Gehäuse mit der Schaltung wird je nach Art der Anwendung entweder im Schlüsselbeinbereich (pektoral) oder im Bauchbereich (abdominal) implantiert. Die in dieser Untersuchung verwendeten Positionen des Neurostimulators bei den verschiedenen Implantationsarten sowie die zugehörigen Kabelverläufe sind in Abb. 3 dargestellt.

Vom Gehäuse ausgehend verläuft ein Elektrodenkabel unter der Haut bis in die Gehirnregion hinein, die stimuliert werden soll. Die Elektrode ist mit einem Mantel aus Silikongummi versehen und besitzt an ihrem Ende vier Kontakte, die einzeln oder gemeinsam zur Anregung genutzt werden können. Um verlässliche Ergebnisse zu erzielen ist es von großer Bedeutung, die Elektrode des Neurostimulators angemessen in die Berechnungen einzubeziehen. Vor allem die Berücksichtigung der Isolierung der Stimulatorelektrode gegenüber dem menschlichen Gewebe ist in jedem Fall notwendig, da sie das Einkoppelverhalten wesentlich beeinflusst. Berechnungen zeigten, dass die Vernachlässigung der Isolierung die eingekoppelte Spannung größenordnungsmäßig um den Faktor zehn niedriger ausfallen lassen kann. 


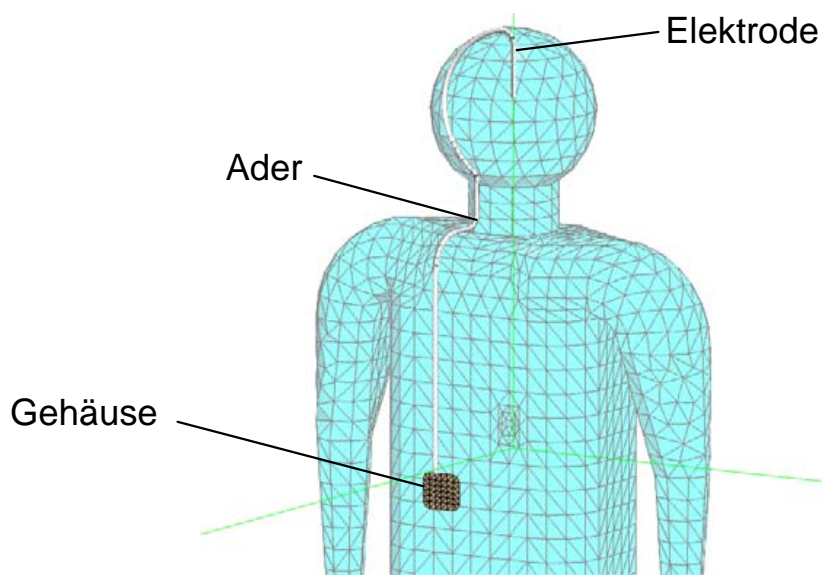

Abbildung 2. Implantierter Neurostimulator bestehend aus Gehäuse und Elektrode.

\section{Untersuchungen und Ergebnisse}

In dieser Untersuchung werden die vom Koagulator abgegebenen Signale als Störursache betrachtet. Dabei werden unterschiedliche Betriebsarten des Gerätes berücksichtigt, um zu „worst-case“-Aussagen zu kommen. Als Störsenke wird hier der Neurostimulator angesehen, dessen Funktionsweise durch die vom Koagulator erzeugte Störspannung an dessen Eingang beeinträchtigt wird. Diese Auswirkung am Eingang des Stimulators wird mit dem Kopplungsmodell beschrieben und ist vom verwendeten Stimulatortyp unabhängig. Die Auswirkung der Störspannung auf den Neurostimulator wird im Verträglichkeitsmodell berücksichtigt und ist nicht Gegenstand der hier angestellten Betrachtungen, die sich auf das Kopplungsmodell konzentriert. Ziel ist es, eine ,worstcase"-Aussage über die durch den Koagulator verursachte Störspannung am Eingang des Neurostimulators auf dessen Elektrode zu erhalten.

Bei der hier dargestellten Untersuchung, die sich mit einem in der Praxis häufig vorkommenden Szenario befasst, werden die Position des Koagulationspunktes im Bauchbereich, die Implantierungsart des Neurostimulators (links bzw. rechts pektoral und links bzw. rechts abdominal) und damit auch die resultierende Länge des Elektrodenkabels, die Länge des Koagulatorkabels sowie die Frequenz (100 kHz bis $100 \mathrm{MHz}$ ) variiert. In Abb. 4 ist beispielhaft ein Berechnungsergebnis für die Implantationsart abdominal rechts, die hier den ,,worst-case“-Fall darstellt, abgebildet.

Es ergeben sich dabei im ,worst-case“-Fall Spannungen in der Größenordnung von einigen Millivolt. Der Fall mit abdominal rechter Implantation stellt bei den durchgeführten Variationen der Berechnungsparameter den ,worst-case“ dar. Dies liegt darin begründet, dass in diesem Fall die Stimulatorelektrode die größte Länge aufweist und somit die größtmögliche Einkopplung stattfindet. Vor allem im oberen Frequenzbereich treten im Spannungsverlauf Resonanzeffekte auf. Die Ursache für diese Effekte liegt in Resonanzen auf dem Koagulatorkabel und auf der Oberfläche der Körpermodelle begründet.

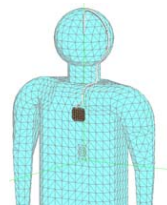

(a)

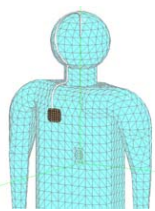

(b)

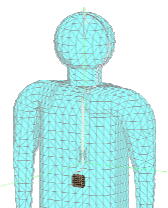

(c)

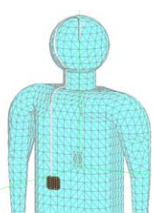

(d)
Abbildung 3. Implantationsarten des Neurostimulators: links (a) und rechts (b) pektoral, sowie links (c) und rechts (d) abdominal.

Störspannung am Einang des Neurostimulators (abdominal rechts)

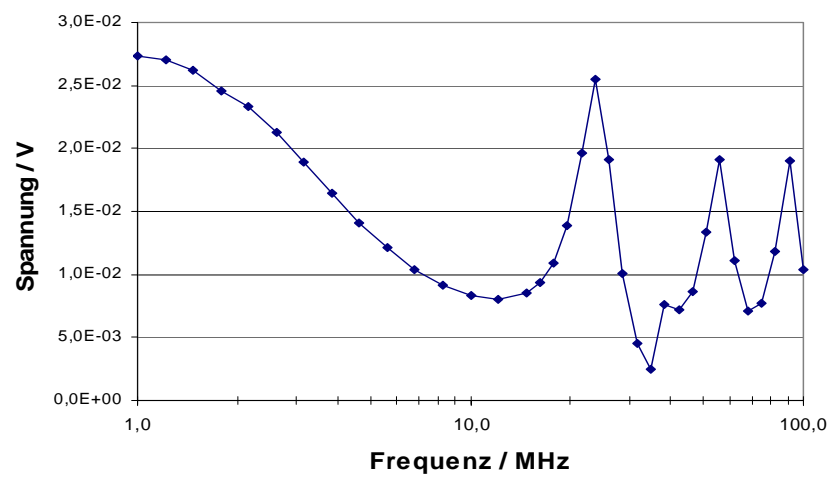

Abbildung 4. Durch den Koagulator verursachte Störspannung auf der Neurostimulatorelektrode am Eingang des Gehäuses über der Frequenz.

\section{Zusammenfassung}

In der hier beschriebenen Untersuchung wird die Verkopplung eines Koagulationsgeräts mit einem implantierten Neurostimulator über den menschlichen Körper betrachtet. Dazu wurde zuerst ein Körpermodell nach DIN mit den entsprechenden elektrischen Eigenschaften des menschlichen Körpers entwickelt und die medizinischen Geräte durch das elektrische Verhalten an ihren Ausgangsklemmen beschrieben. Die durch den Koagulator am Eingang des Neurostimulators auf dessen Elektrode verursachte Störspannung ist beispielhaft aufgeführt und es werden an Hand dieser die abgeleiteten Ergebnisse verdeutlicht. Das beschriebene Kopplungsmodell erlaubt es nun, eine „,worst-case"-Aussage über die Verkopplung der beiden Medizingeräte zu treffen.

Danksagung. Die Autoren danken der Deutschen Forschungsgemeinschaft für die Finanzierung des Projektes und den Firmen Erbe Elektromedizin, Philipps Medical Systems und Aesculap für die freundliche Unterstützung und die zahlreichen Anregungen. 


\section{Literatur}

Deutsche Norm: Körpermaße des Menschen; Werte, DIN 33 402, Teil 2, 1-33, 1986.

EM Sorfware \& Systems: FEKO User's Manual, Suite 4.1, 2003.

Gabriel, C.: Compilation of the Dielectric Properties of Body Tissues at RF and Microwave Frequencies, Brooks Air Force, Techn. Report AL/OE-TR-1996-0037, 1996.

Landstorfer, F. M., Geisbusch, L., Jakobus, U., Maier, M., Ruoß, H.O., Spreitzer, W., und Waldmann, J.: Development of a model describing the coupling between electrodes of cardiac pacemakers and transmitting antennas in their close vicinity in the frequency range from $50 \mathrm{MHz}$ to $500 \mathrm{MHz}$, Forschungsgemeinschaft Funk, Final Report, sec. ed., 1999.
Italian National Research Council: Calculation of the Dielectric Properties of Body Tissues in the frequency range $10 \mathrm{~Hz}-$ $100 \mathrm{GHz}$, http://safeemf.iroe.fi.cnr.it/tissprop/htmlclie/htmlclie. htm, 1997.

Koritke, J. G. and Sick, H.: Atlas of Sectional Human Anatomy, Urban and Schwarzenberg, Baltimore, 1988. 